AperTO - Archivio Istituzionale Open Access dell'Università di Torino

\title{
The 4D pixel challenge
}

\section{This is the author's manuscript}

Original Citation:

Availability:

This version is available http://hdl.handle.net/2318/1636961

since 2017-05-24T21:06:23Z

Published version:

DOI:10.1088/1748-0221/11/12/C12016

Terms of use:

Open Access

Anyone can freely access the full text of works made available as "Open Access". Works made available under a Creative Commons license can be used according to the terms and conditions of said license. Use of all other works requires consent of the right holder (author or publisher) if not exempted from copyright protection by the applicable law. 


\title{
The 4D pixel challenge
}

\author{
N. Cartiglia ${ }^{a}$ R. Arcidiacono ${ }^{a, c}$ A. Bellora ${ }^{b}$ F. Cenna ${ }^{a, b}$ R. Cirio ${ }^{a, b}$ S. Durando ${ }^{b}$ A. Fadavi ${ }^{b, f}$ \\ M. Ferrero ${ }^{a, b}$ Z. Galloway ${ }^{e}$ B. Gruey ${ }^{e}$ P. Freeman ${ }^{e}$ M. Mandurrino $^{a, d}$ V. Monaco $^{a, b}$ R. \\ Mulargia $^{a, b}$ M. Obertino ${ }^{a, b}$ F. Ravera ${ }^{a, b}$ R. Sacchi ${ }^{a, b}$ H.F-W.Sadrozinski ${ }^{e}$ A. Seiden ${ }^{e}$ V.

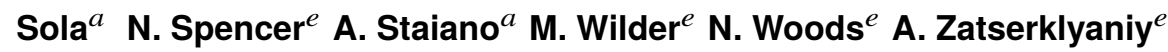 \\ ${ }^{a}$ INFN Torino \\ ${ }^{b}$ Università di Torino, Torino, Italy \\ ${ }^{c}$ Università del Piemonte Orientale, Novara, Italy \\ ${ }^{d}$ Politecnico di Torino, Torino, Italy \\ e'Santa Cruz Institute for Particle Physics UC Santa Cruz, CA, 95064, USA \\ ${ }^{f}$ Universita' di Sabzevar, Iran \\ E-mail: cartiglia@to.infn.it
}

AвSTRACT: Is it possible to design a detector able to concurrently measure time and position with high precision? This question is at the root of the research and development on silicon sensors presented in this contribution. Silicon sensors are the most common type of particle detectors used for charged particle tracking, however their rather poor time resolution limits their use as precise timing detectors. A few years ago we have picked up the gantlet of enhancing the remarkable position resolution of silicon sensors with an equally precise timing capability. I will be presenting our results in the following pages.

KeYwords: Only keywords from JINST's keywords list please

${ }^{1}$ Corresponding author 


\section{Contents}

1 Setting the stage 1

2 The effect of timing information 2

3 Time-tagging detectors $\quad 4$

3.1 Jitter 5

3.2 Choice of Time-walk correction circuits 5

3.3 The effect Landau fluctuations: time walk and Landau noise 5

3.4 Signal Distortion: non uniform weighting field and not saturated drift velocity 6

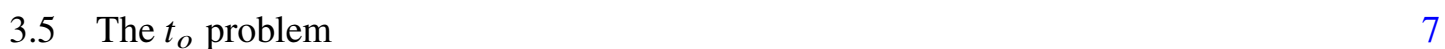

3.6 Interplay of signal rise time, detector capacitance and read-out input impedance $\quad 7$

3.7 Roadmap 8

4 Design of a 4D tracker using Ultra-Fast Silicon Detectors $\quad 8$

4.1 LGAD - Low-Gain Avalanche Diodes $\quad 8$

4.2 The effect of charge multiplication 9

$\begin{array}{lll}4.3 & \text { Landau noise in UFSD sensors } & 11\end{array}$

$\begin{array}{lll}\text { 4.4 Shot Noise in UFSD sensors } & 11\end{array}$

4.5 Radiation effect in UFSD sensors 12

5 Productions and Performances of Ultra-Fast Silicon Sensors 14

$\begin{array}{ll}\text { 5.1 Results from 50-micron thick UFSD } & 14\end{array}$

6 Summary 14

$\begin{array}{ll}\text { 6.1 Optimum Sensor Design } & 15\end{array}$

\section{Setting the stage}

A recent development in silicon detector technology is the capability of adding low controlled gain into an otherwise standard silicon sensor [1]. This new type of silicon sensor, the so called Low Gain Avalanche Diodes (LGAD), promises to significantly enhance the capability to measure track arrival times, leading to a dramatic improvement in the capability of silicon arrays. The goal is to simultaneously maintain the high granularity for spatial measurement and the capability for high rate data collection while making very good time measurements [2]. In fact the time measurement requires very short duration signals allowing even larger data rates than conventional silicon sensors. Not all sensors geometries, thicknesses or gain values are suitable for precise timing measurements: we call Ultra-Fast Silicon Detectors (UFSD) those LGAD sensors optimized for timing measurements [3]. 


\section{The effect of timing information}

The inclusion of timing information in the structure of a recorded event has the capability of changing the way we design experiments, as this added dimension dramatically improves the reconstruction process. The most obvious simplification is that only time-compatible hits are used in the pattern recognition phase, discarding those hits that cannot be associated to a track due to an excessive time difference.

Depending on the type of sensors that will be used, timing information can be available at different stages in the reconstruction of an event. The most complete option is that timing is associated to each point of the track: in this case the electronics needs to be able to accurately measure the time of the hit in each pixel. This option is indeed quite difficult to achieve, due to the massive increase of power consumption that such architecture would require. Nevertheless, as the potential gain that this option offers in terms of performances is the largest, we have set this option as our final goal. Figure 1 schematically shows the effect of having timing information for each hit. This capability has been exploited in the Retina project [4].

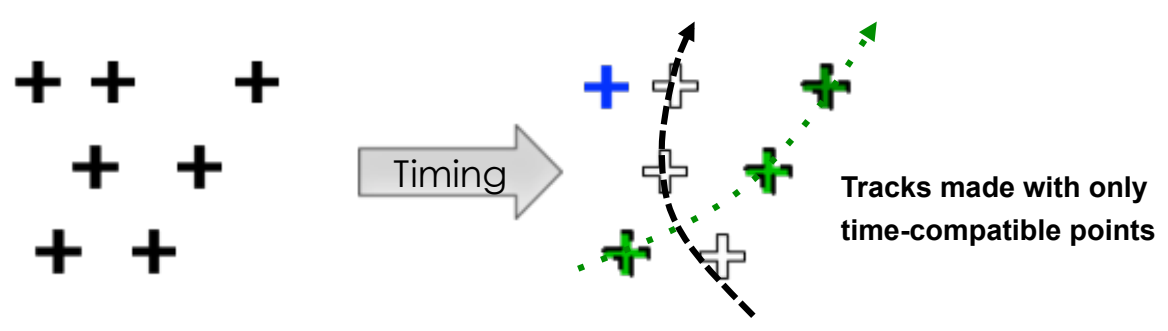

Figure 1. Effect of the inclusion of timing information at the hit level: only time-compatible points are associated to a track.

A very interesting option to deal with the increased power consumption mentioned above it is to reduce the granularity of the timing information, moving the timing sensors outside the volume of the tracker. This specific idea has been explored by ATLAS [5] in order to improve Level 1 trigger decision. The timing information at the trigger level can play a key role in avoiding the saturation of the first level trigger (L1) bandwidth with fake events: as timing information can be obtained quite fast, its use in L1 decisions will allow distinguishing events with the same topology but originating in either one or many collisions. Figure 2 shows two examples of the added value of timing information at the trigger level: on the top part, the topology without timing information indicates a 3 -jet event, while on the right the 3 jets are correctly assigned to a 2 -jet plus missing $E_{T}$ event (that is an event where the detector energies do not balance indicating an unidentified penetrating particle) plus an overlapping jet from another event. The bottom part of Figure 2 illustrates how timing information is used to identify if a forward jet is actually due to a unique physics process (as in WW fusion for example), or it is just due to the random clustering of tracks coming from unrelated scattering.

To retain the full power of timing information at the event reconstruction level, i.e. to maximize the precision of the reconstructed kinematical quantities, it is actually sufficient assigning a time to each track. This path is much easier than assigning time to each hit, as it can be done 
a)

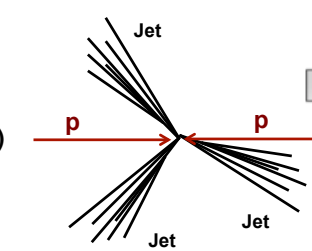

3-Jet event?

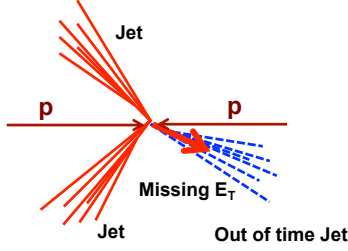

2-Jet and Missing $\mathrm{E}_{\mathrm{T}}$ event

b)

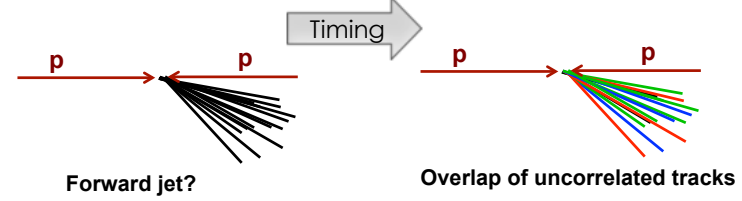

Figure 2. Effect of timing information of the topology of the event: a) a 3-jet event is re-interpreted as a 2jet plus missing $E_{T}$ event plus an overlapping jet from different event, b) a forward jet is correctly recognized to be due to the overlap of uncorrelated

with a single dedicated timing layer either inside or outside the tracker volume. This path is now under consideration in the CMS upgrade [6], as it would retain a large part of the benefits of 4-D reconstruction, without the burden of redesigning the tracker system. Figure 3 shows schematically 3 situations where the timing information is crucial to the correct definition of the event topology: a) two overlapping events are recognized as one event with missing $E_{T}$ plus a minimum bias event, b) in $H \rightarrow \gamma \gamma$ events, the timing of the two photons identify a small region of space where the correct vertex is located: vertex timing allows making the correct match, c) secondary tracks can be correctly assigned to their vertex.

a)
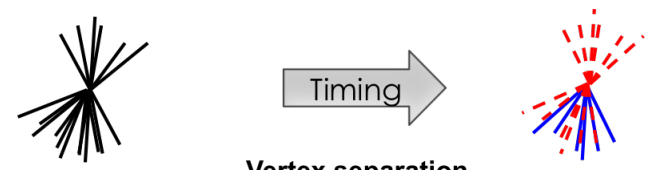

Vertex separation

b)
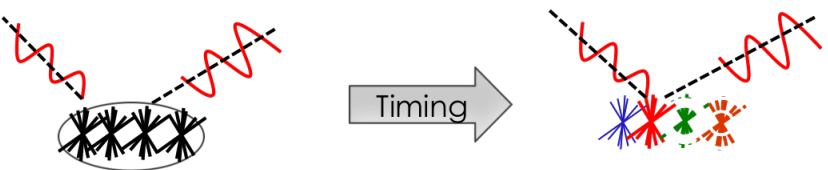

$\gamma \gamma$ vertex

c)

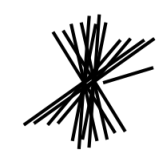

\section{Timing}

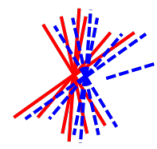

Displaced track assignment

Figure 3. The knowledge of track-timing allows assigning correctly each track to the proper vertex: a) overlapping events, b) matching of the $H \rightarrow \gamma \gamma$ vertex, c) heavy flavor decays. 
Considering a specific situation, at HL-LHC [7] the number of events per bunch crossing will be of the order of 150-200, with an average distance between vertexes of 500 micron and a timing rms spread of $150 \mathrm{ps}$. Considering a vertex separation resolution of 250-300 micron along the beam direction (present resolution for CMS and ATLAS), there will be 10-15\% of vertexes composed by two events. Without the possibility to separate these vertexes using timing information, this overlap will cause a degradation in the precision of the reconstructed variables, and lead to loss of events. We can therefore conclude that timing information at HL-LHC is equivalent of having additional luminosity.

\section{Time-tagging detectors}

Figure 4 shows the main components of a time-tagging detector. For a pioneering article on timing see [8] while for an up-to-date review of current trends in electronics see [9]. The sensor, shown as a capacitor with a current source in parallel, is read-out by a pre-amplifier that shapes the signal. The pre-amplifier's output is then compared to a fixed threshold to determine the time of arrival. In the following we will use this simplified model to explore the timing capabilities of various detectors, while for the moment we will not consider more complex and space-consuming approaches such as waveform sampling. It is interesting to note that averaging multiple $(\mathrm{N})$ measurements at different threshold does not lead to an improvement in the time resolution proportional to $1 / \sqrt{N}$ since the points are strongly correlated.

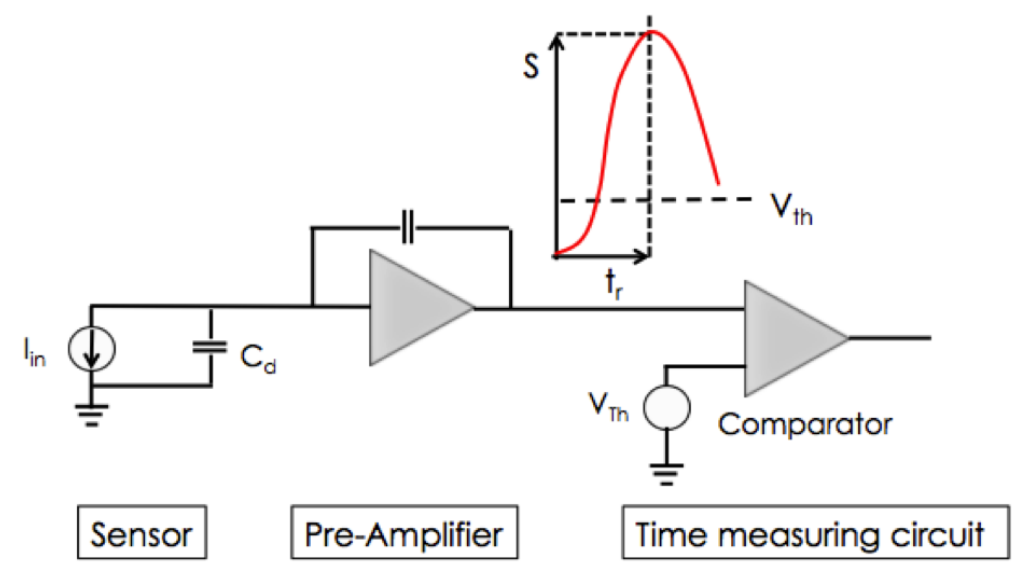

Figure 4. Main components of a time-tagging detectors. The time is measured when the signal crosses the comparator threshold.

The time resolution $\sigma_{t}$ can be expressed as the sum of several terms: (i) Jitter, (ii) Landau shape variation, (iii) Time Walk, (iv) signal distortion, and (v) TDC binning:

$$
\sigma_{t}^{2}=\sigma_{\text {Jitter }}^{2}+\sigma_{\text {Landau Noise }}^{2}+\sigma_{\text {Time Walk }}^{2}+\sigma_{\text {Distortion }}^{2}+\sigma_{T D C}^{2} .
$$

For the sake of clarity, in the following we will assume two simplifications: 
- we consider the effect of time walk (see [3] for details) compensated by an appropriate electronic circuit (either Constant Fraction Discriminator or Time over Threshold), see Section 3.2. With this assumption, the effect of Landau variations in signal amplitude are compensated, but not that of shape variation (in eq. 3.1 this second contribution is indicated as Landau noise, $\sigma_{\text {Landau Noise }}^{2}$ ).

- the contribution of TDC binning to be below $10 \mathrm{ps}$ and therefore it will be ignored.

\subsection{Jitter}

The jitter term represents the time uncertainty caused by the early or late firing of the comparator due to the presence of noise on the signal, it is directly proportional to the noise $N$ of the system and it is inversely proportional to the slope of the signal around the value of the comparator threshold. Assuming a constant slope we can write $d V / d t=S / t_{r}$ and therefore:

$$
\sigma_{J}=\frac{N}{d V / d t}=\frac{t_{r}}{S / N}
$$

\subsection{Choice of Time-walk correction circuits}

Time-walk, the unavoidable process by which larger signals cross a given threshold earlier than smaller ones, needs to be corrected by an appropriate electronic circuit. The three most common solutions are illustrated in Figure 5: (a) Constant Fraction Discriminator (CFD), which sets the time of arrival of a particle when the signal reaches a given fraction of the total amplitude, (b) Time over Threshold (ToT), that uses two time points to evaluated the amplitude of the signal, and apply a correction amplitude-dependent to the first time point $t_{1}$ and (c) Multiple Samplings (MS), where the signal is sampled multiple times, and a fit is used to define the particle time. CFD and ToT are simpler solutions, and they can be implemented per pixel within the read-out chip. MS is instead a rather complex algorithm as it requires the full digitization of the signal: this solution gives the best performance, but it can be used only for systems with a limited number of pixels as it needs a fair amount of computing power. The choice among these 3 possibilities is a system decision that needs to be taken considering all other aspects of the experiment.
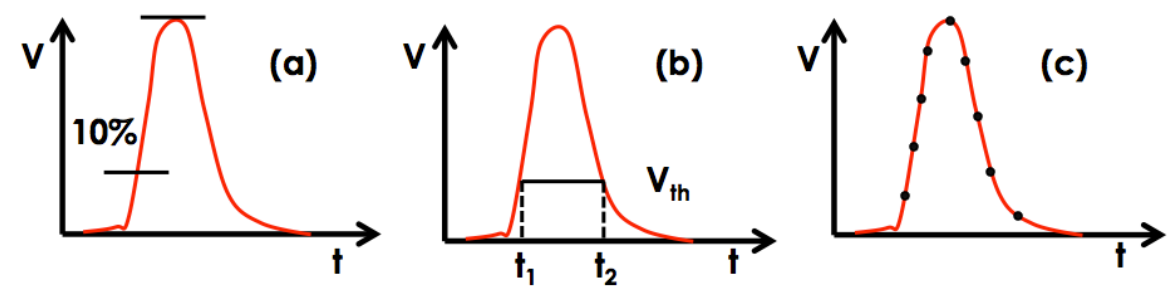

Figure 5. Time-walk correction techniques: (a) Constant Fraction Discriminator, (b) Time Over Threshold, (c) Multiple Samplings.

\subsection{The effect Landau fluctuations: time walk and Landau noise}

The ultimate limit to signal uniformity is given by the physics governing energy deposition: the charge distribution created by an ionizing particle crossing a sensor varies on an event-by-event 
basis. These variations not only produce an overall change in signal magnitude, which is at the root of the time walk effect (that we assumed perfectly corrected by electronics), but also produce a more irregular current signal (Landau noise). The left part in Figure 6 shows 2 examples of the simulated [10] energy deposition by a minimum ionizing particle, while the right part the associated generated current signals and their components. As the picture shows, the variations are rather large and they can severely degrade the achievable time resolution. There are two ways to mitigate this effect: (i) integrating the output current over times longer than the typical spike length and (ii) using thin sensors, as their steeper signal is more immune to signal fluctuations.
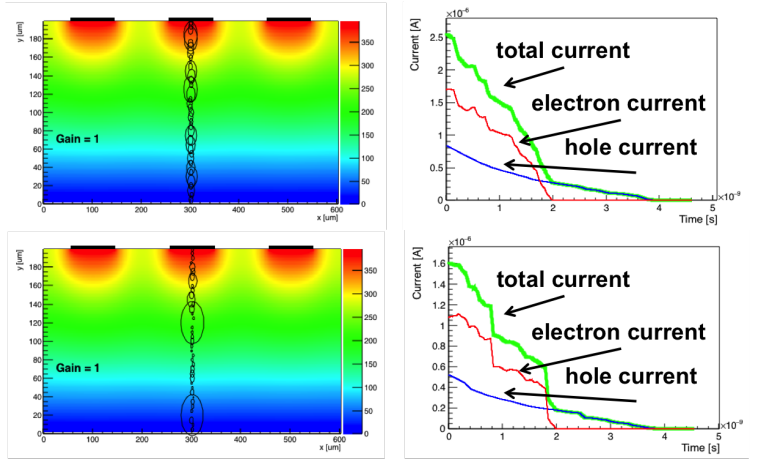

Figure 6. Energy deposits in a silicon detectors and the corresponding current signals.

\subsection{Signal Distortion: non uniform weighting field and not saturated drift velocity}

In every particle detector, the shape of the induced current signal can be calculated using Ramo's [11] theorem that states that the current induced by a charge carrier is proportional to its electric charge $q$, the drift velocity $v$ and the weighting field $E_{w}$, equation (3.3):

$$
i \propto q v E_{w} .
$$

This equation indicates several key points in the design of sensors for accurate timing, shown in Figure 7:

- the drift velocity needs to be constant throughout the volume of the sensor. Non-uniform drift velocity induce variations in signal shape as a function of the hit position, Figure 7 a), spoiling the overall time resolution. The easiest way to obtain uniform drift velocity throughout the sensor is to have an electric field high enough to move the carriers with saturated drift velocity.

- the weighting field $E_{w}$ represents the capacitive coupling of a charge $e$ to the read-out electrode. If this coupling depends on the impinging particle position along the strip pitch, Figure $7 \mathrm{~b}$ ), the signal shape would be different depending on the hit position, spoiling the time resolution. Strips need to have the width very similar to the pitch, and larger than the signal thickness: width $\sim$ pitch $»$ thickness. 


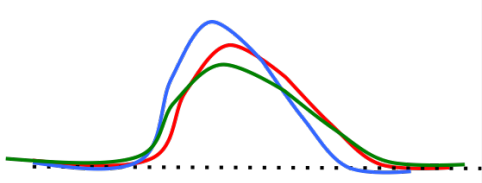

a)

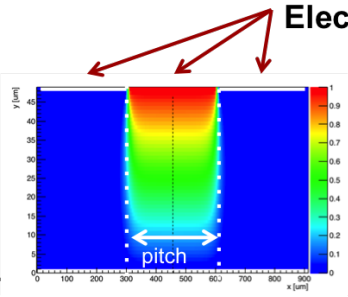

Wide Strip

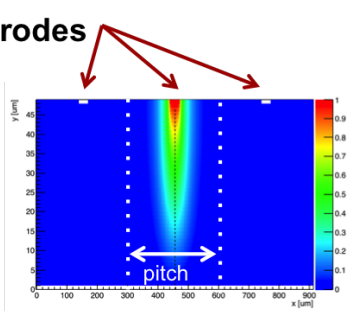

Thin Strip

b)

Figure 7. a) Effect of velocity variation on the signal shape b) Weighting field for two configurations: (left) Wide strips, (right) Thin strips.

\subsection{The $t_{o}$ problem}

In systems where the weighting field is not constant over the sensor volume there is an additional source of time uncertainties: before the particle signal can become visible, the charge carriers have to drift from the impact point to the region of high weighting field. This effect is shown schematically in Figure 8. In silicon, electrons with saturated velocity move at about $1 \mu \mathrm{m}$ per 10 picosecond: this effect can easily become the dominant source of time uncertainties.

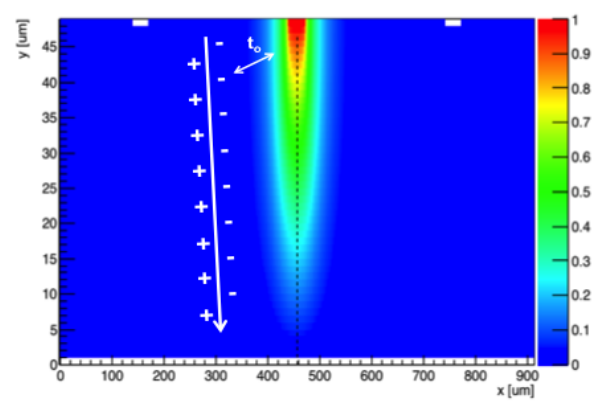

Figure 8. A non-uniform weighting field causes an additional source of time uncertainties due to the drift time from the impact point to the region of high weighting field.

\subsection{Interplay of signal rise time, detector capacitance and read-out input impedance}

The charges collected on the read-out electrode of the sensor move to the input of the read-out electronics with a time constant $\tau$ given by the product of the detector capacitance $C_{d e t}$ and the read-out input impedance $R_{i n}: \tau=R_{i n} C_{\text {det }}$, Figure 9.

In order not to slow down the signal rise time, $\tau$ has to be shorter or, at most, of the same order of the signal current rise time, $t_{C u r}$. This constrain is strongly linking sensor and electronics designs, as the electronics should be such that it does not slow down very fast input signals. The signal rise time at the output of the pre-amplifier determines $d V / d t$ and therefore is of the utmost importance in time-tagging detectors. We can identify 3 distinct effects that influence the signal 


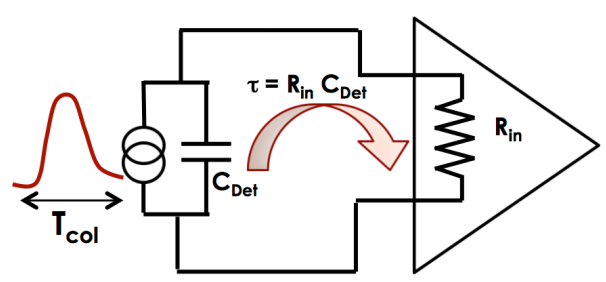

Figure 9. Interplay of the signal rise time, detector capacitance and read-out input impedance.

slew rate at the comparator input, eq. 3.4: (i) the signal rise time $t_{C u r}$ that depends on the properties of the sensor, (ii) the $t_{R C}=R_{i n} C_{d e t}$ time constant, and (iii) the amplifier rise time $t_{A m p}$, which should be matched to $t_{C u r}$ and $t_{R C}$ to minimize the noise of the amplifier:

$$
t_{\text {rise }}=\sqrt{t_{\text {Cur }}^{2}+t_{R C}^{2}+t_{A m p}^{2}} \text {. }
$$

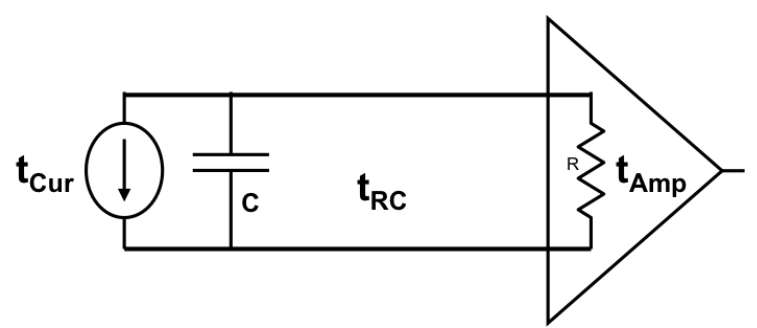

Figure 10. Schematic representation of the 3 main time constants affecting the signal rise time at the preamplifier output.

\subsection{Roadmap}

The integration of time-tagging capabilities into a position sensor produces a steep increase in system complexity. Part of this complexity can be addressed by smart architectures, new technological nodes (for example $65 \mathrm{~nm}$ ) allowing higher circuit densities and new chip designs. However the present bottlenecks inherent to hybrid systems, having sensors and electronics built on separated substrates, will ultimately limit the complexity and drive the cost. Most likely the real turning point of 4D tracking will happen when monolithic technology will be mature enough to allow integrating the sensor and the electronics in the same substrate, reducing interconnections and keeping the capacitance of each sensor low. As we are deciding now (2016) the choices for HL-LHC (2025), this evolution will not be used at HL-LHC, but it will probably appear first in smaller experiments, and then be used on larger scales.

\section{Design of a 4D tracker using Ultra-Fast Silicon Detectors}

\subsection{LGAD - Low-Gain Avalanche Diodes}

Standard silicon detectors can be used in timing applications, provided the sensor geometry is appropriate. Currently the NA62 experiment [12] is employing a track-timing detector, the so 
called Gigatracker, that uses 200-micron thick sensors with 300x300 micron pixels. The expected time resolution is around $\sigma_{t} \sim 150 \mathrm{ps}$. Recently [13], employing an extremely low noise new circuit, a resolution of $\sigma_{t} \sim 105$ ps has been reached using a 100-micron thick, $4 \mathrm{~mm}^{2}$ square pad sensor. Standard silicon sensors have therefore the capability of reaching good time resolutions, however it is rather difficult to reach resolutions better than $\sigma_{t} \sim 80-100$ ps given their small signal. The natural evolution of this problem is therefore to manufacture silicon sensors with a larger output signal: the Low-Gain Avalanche Diodes (LGAD).

LGAD is a new concept in silicon detector design, merging the best characteristics of standard silicon sensors with the main feature of APDs. The overarching idea is to design silicon detectors with signals that are a factor of 10 higher than those of standard sensors, however without the problems connected with the APD high gain [14-18].

Charge multiplication in silicon sensors happens when the charge carriers are in electric fields of the order of $E \sim 300 \mathrm{kV} / \mathrm{cm}$. Under this condition the electrons (and to less extent the holes) acquire sufficient kinetic energy that are able to generate additional $\mathrm{e} / \mathrm{h}$ pairs. A field value of 300 $\mathrm{kV} / \mathrm{cm}$ is not reachable applying an external voltage $V_{\text {Bias }}$ without causing electrical breakdown, but it is obtained by implanting an appropriate charge density that locally generates very high fields $\left(N_{D} \sim 10^{16} / \mathrm{cm}^{3}\right)$. The gain has an exponential dependence on the electric field $N(l)=N_{o} e^{\alpha(E) l}$, where $\alpha(E)$ is a strong function of the electric field and $l$ is the path length inside the high field region. The additional doping layer present at the $n-p$ junction in the LGAD design, Figure 11, generates the high field necessary to achieve charge multiplication.
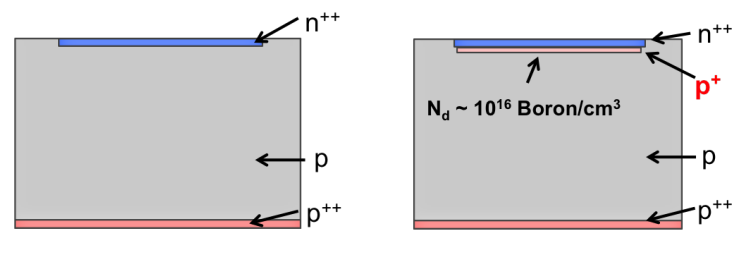

Traditional Silicon Diode

Low Gain Avalanche Diode

Figure 11. Schematic of a traditional silicon diode (left) and of a Low-Gain Avalanche Diode (right).

According to our simulation program Weightfield2 ${ }^{1}$ (WF2), LGAD have the potentiality of replacing standard silicon sensors in almost every application, with the added advantage of having a large signal $d V / d t$ and therefore being able to measure time accurately. In the following, we will use the name of "Ultra-Fast Silicon detectors" (UFSD) to indicate LGAD sensors optimized for timing performances.

\subsection{The effect of charge multiplication}

Using WF2 we can simulate the output signal of UFSD sensors as a function of many parameters, such as the gain value, sensor thickness, electrode segmentation, and external electric field. Figure 12 shows the simulated current, and its components, for a 50-micron thick detector. The initial electrons (red), drifting toward the $\mathrm{n}++$ electrode, go through the gain layer and generate additional

\footnotetext{
1Open source code, link at cern.ch/nicolo
} 
e/h pairs. The gain electrons (violet) are readily absorbed by the cathode while the gain holes (light blue) drift toward the anode and they generate a large current.

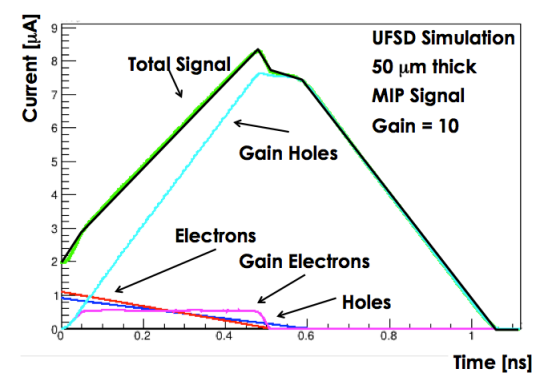

Figure 12. UFSD simulated current signal for a 50-micron thick detector.

The gain dramatically increases the signal amplitude, producing a much higher slew rate. The value of the current generated by a gain $G$ can be estimated in the following way: (i) in a given time interval $d t$, the number of electrons entering the gain region is $75 v d t$ (assuming $75 \mathrm{e} / \mathrm{h}$ pairs per micron); and (ii) these electrons generate $d N_{\text {Gain }} \propto 75 \mathrm{v} d t G$ new e/h pairs. Using again Ramo's theorem, the current induced by these new charges is given by:

$$
d i_{\text {Gain }}=d N_{\text {Gain }} q v_{\text {sat }} \frac{k}{d} \propto \frac{G}{d} d t,
$$

which leads to the following expression for the slew rate:

$$
\frac{d i_{\text {Gain }}}{d t} \sim \frac{d V}{d t} \propto \frac{G}{d} .
$$

Equation (4.2) demonstrates a very important feature of UFSD: the slew rate increase due to the gain mechanism is proportional to the ratio of the gain value over the sensor thickness $(G / d)$, therefore thin detectors with high gain provide the best time resolution. Specifically, the maximum signal amplitude is controlled only by the gain value, while the signal rise time only by the sensor thickness, Figure 13.

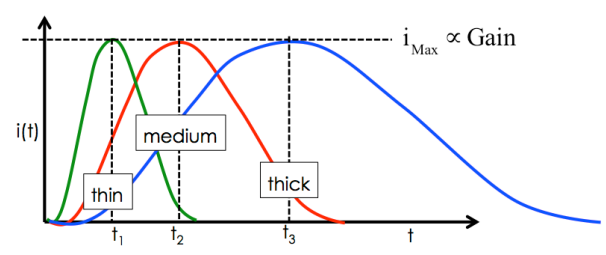

Figure 13. In UFSD the maximum signal amplitude depends only on the gain value, while the signal rise time only on the sensor thickness: sensors of 3 different thicknesses (thin, medium, thick) with the same gain have signals with the same amplitude but with different rise time.

Using WF2 we have cross-checked this prediction simulating the slew rate for different sensors thicknesses and gains, Figure 14: the slew rate in thick sensors, 200- and 300-micron, is a factor of $\sim 2$ steeper than that of traditional sensors, while in thin detectors, 50- and 100-micron thick, the slew rate is 5-6 times steeper. 


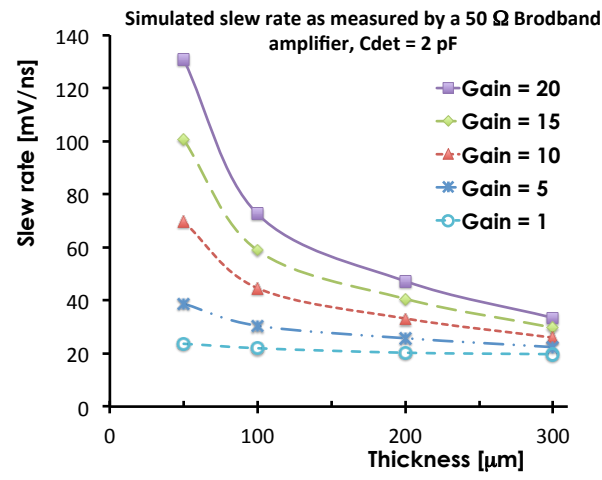

Figure 14. Simulated UFSD slew rate as a function of gain and sensor thickness. Thin sensors with even moderate gain $(10-20)$ achieve a much higher slew rate than traditional sensors (gain $=1)$.

\subsection{Landau noise in UFSD sensors}

With WF2 we have studied in details the effect of Landau noise on time resolution, Figure 15. The picture shows several important effects: (i) Landau noise sets a physical limit to the precision of a given sensor which is of the order of $20 \mathrm{ps}$ in thin sensors, and much larger for thicker sensors, (ii) Landau noise is minimized by setting the comparator threshold as low as possible, (iii) thin detectors are less prone to Landau noise.

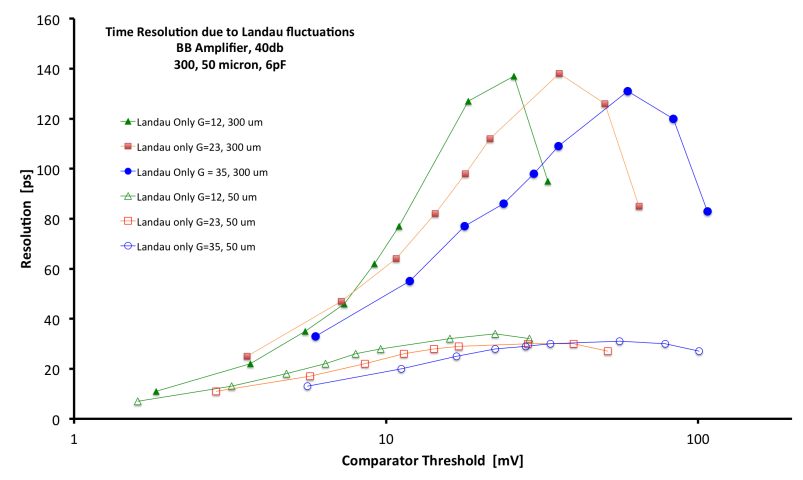

Figure 15. Effect of Landau noise on time resolution. Best results are obtained for thin sensors, while the effect of gain on Landau noise is less important.

\subsection{Shot Noise in UFSD sensors}

Shot noise arises when charge carriers cross a potential barrier, as it happens in silicon sensors. In sensors such as UFSD or APD this effect is enhanced by the gain and for this reason shot noise can be the dominant source of noise for this type of detectors. As shown in Figure 16 a), the 
sensor leakage current is the sum of two components: (i) surface current, that does go through the multiplication layer, and (ii) bulk current, that is multiplied by the gain mechanism.

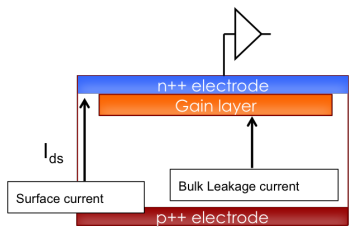

a)

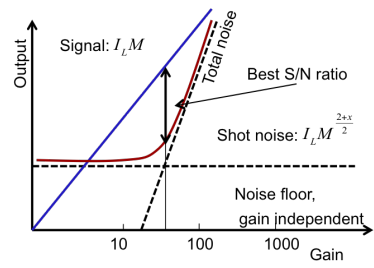

Figure 16. a) Sketch of the shot noise mechanism in sensors with internal gain: bulk current is multiplied by the gain, while surface current is not. b) For increasing gain, shot noise increases faster than the signal.

When carriers undergo multiplication, there is an additional mechanism that enhance shot noise: multiplication is a statistical process, therefore some carries multiply more than others, causing an increase in noise, the so called excess noise factor, ENF. This effect is in addition to the increase in noise due to the gain value that simply multiply the leakage current. ENF causes a very peculiar effect: in device with gain, as the gain increases the ratio signal/noise (S/N) becomes smaller since shot noise increases faster than the signal, Figure $16 \mathrm{~b}$ ). In order to obtain a beneficial effect from the gain mechanism is therefore necessary to have a gain value small enough (gain $\leq$ 20) that the signal increases while the noise increment is small enough to be in the shadow of the electronic noise floor. Shot noise is normally smaller than the electronic noise floor for un-irradiated sensors, but it can become the dominant source of noise for irradiated detectors. Figure 17 shows the value of shot noise for a $4 \mathrm{~mm}^{2} 50$-micron thick silicon sensor, assuming a 2-ns long integration time. In the plots the electronic noise is assumed to be 450 ENC. Figure 17 a) demonstrates the dramatic effect of gain on shot noise, while Figure $17 \mathrm{~b}$ ) the effect of temperature (leakage current decreases a factor of 2 every 7 degrees).

Figure 17 demonstrates that shot noise can become the most important source of noise for irradiated sensors with gain, and suggests that low gain and low temperature can keep this effect under control. Additionally, shot noise can be kept under control by keeping small the volume of the each pixel/strip, as leakage current depends on the overall volume: $I_{\text {Leak }}=\alpha * \Phi * V$, with $\alpha=3 \cdot 10^{-17} \mathrm{~cm}^{-1}$ and $\Phi$ the particle fluence in $n_{e q} / \mathrm{cm}^{2}$.

\subsection{Radiation effect in UFSD sensors}

Radiation damage causes three main effects: (i) decrease of charge collection efficiency, (ii) increase of leakage current, and (iii) changes in doping concentration.

Decrease of charge collection efficiency: Charge collection efficiency (CCE) measures the fraction of charge carriers that are not trapped by lattice defects. This fraction decreases with increasing radiation levels (with differences depending on the type of silicon and type of irradiation) and with increasing drift length. As a rule of thumb, for irradiation levels around $10^{15} n_{e q} / \mathrm{cm}^{2}$, the mean free path in silicon is around 50 micron. Figure 18 shows the simulated [19] signal changes as a function of radiation level for a 50-micron thick sensor: the effect is rather small up to a fluency 

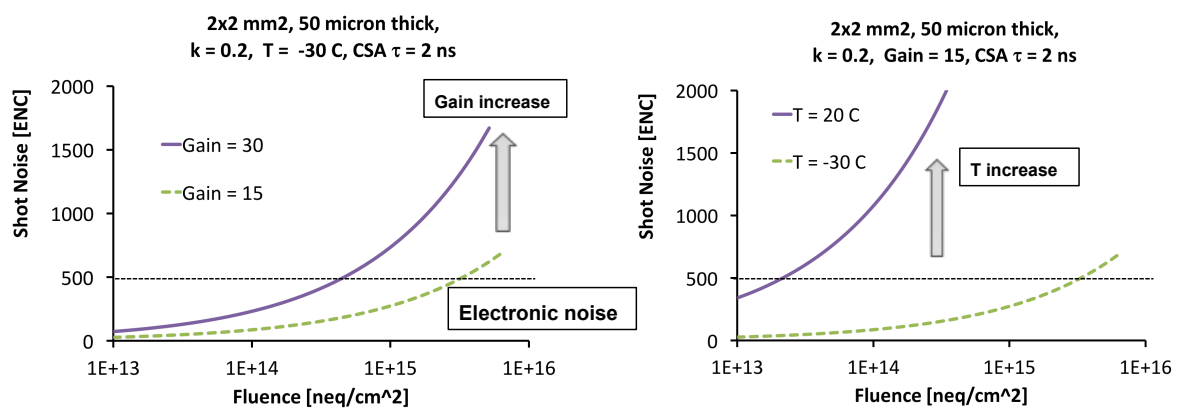

Figure 17. a) Shot noise increase as a function of fluence for two different gain values. b) Shot noise increase as a function of fluence for two different temperature values

of $10^{15} n_{e q} / \mathrm{cm}^{2}$, and it becomes important above a fluency of $5 \cdot 10^{15} n_{e q} / \mathrm{cm}^{2}$. Interestingly, the initial edge of the signal, used for timing, it is not affected much.

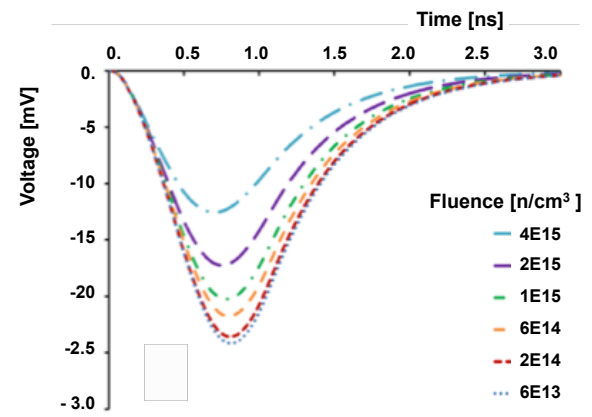

Figure 18. Signal change in a 50-micron silicon sensor as a function of irradiation levels (the only effect considered in this plot is charge trapping).

Increase of leakage current: An increase in leakage current causes two important effects: (i) a larger noise, as explained in Section 4.4, and (ii) a change in the apparent doping concentration. This second effect can be quite important, and it is presently under intense study.

Changes in doping concentration: UFSD sensors have shown a decrease of gain values for fluences above $10^{14} n_{e q} / \mathrm{cm}^{2}$, with a complete disappearance of the gain at $10^{15} n_{e q} / \mathrm{cm}^{2}$. This effect has not been understood yet, but there are two possible explanations: (i) an inactivation of acceptors due to radiation defects [20], and (ii) a dynamic reduction of the gain layer doping due to charge trapping. There are currently three research paths in the investigation of gain reduction, aiming at establishing a radiation hard design for UFSD: (i) change the dopant of the p-type gain layer from Boron to Gallium, as Gallium has been shown to be more radiation resistant, (ii) development of very thin sensors, to decrease the amount of leakage current and therefore trapping, and (iii) doping of the gan layer using Carbon [21]. 


\section{Productions and Performances of Ultra-Fast Silicon Sensors}

The first publication containing measurements of LGAD sensors was been presented in 2014 by the Centro Nacional de MicroelectrUnica (CNM) Barcelona [15] while the first production of thin UFSD $(50 \mu \mathrm{m})$ by CNM was presented in 2016 [22]. First beam test results on thin UFSD manufactured by CNM have been obtained in 2016 [23]. The Fondazione Bruno Kessler (FBK) has also designed [16] and produced LGAD sensors, up to now only 300-micron thick; first FBK production of thin LGAD is expected in early 2017. In the past 3 years CNM has manufactured a variety of LGAD designs, exploring different substrates (float zone (FZ), silicon-on-insulator (SoI), epitaxial (epi) with high and medium resistivity), reaching a well-controlled manufacturing capability. FBK has manufactured a single run of very high quality, exploring traditional LGAD design, segmented p-side read-out and AC coupling read-out.

\subsection{Results from 50-micron thick UFSD}

In this section we report on the results obtained in a beam test at CERN with $\pi$-mesons with a momentum of $180 \mathrm{GeV} / \mathrm{c}$. Several $1.2 \times 1.2 \mathrm{~mm}^{2}$ UFSD sensor read-out by a fully custom broadband amplifier and a trigger board comprising of a SiPM coupled to a quartz bar were used [23]. This beam test, coupled with complementary laser measurements performed in our laboratories, provides the opportunity to perform detailed studies of the mechanisms governing UFSD time resolution and to compare these measurements to our simulation.

The left side of Figure 19 shows typical beam test signals, and the right side a comparison between data and WF2, demonstrating the capability of WF2 reproduce the UFSD beam test signals accurately. The signals are very fast, with low noise and large slew rate, ideal for timing studies. The time resolution of each sensor and that of the SiPM has then been obtained from the time differences between pairs of UFSD and between each UFSD and the SiPM, yielding two values for each UFSD. The time resolution of combined UFSD has been evaluated as the difference between the average time of two or three UFSD and the SiPM, Table 1. The results of Table 1 agree well with the expected $\sigma(N)=1 / \sqrt{N}$ behaviour, demonstrating that the 3 sensors are of equal high quality. The timing resolution of a single UFSD is measured to be $35 \mathrm{ps}$ for $200 \mathrm{~V}$ bias and $26 \mathrm{ps}$ for $240 \mathrm{~V}$ bias. A system of three UFSD has a measured timing resolution of $20 \mathrm{ps}$ for a bias of $200 \mathrm{~V}$, and $15 \mathrm{ps}$ for a bias of $240 \mathrm{~V}$.

Table 1. Timing resolution for single $(\mathrm{N}=1)$, doublet $(\mathrm{N}=2)$ and triplets $(\mathrm{N}=3)$ of UFSD at bias voltages of $200 \mathrm{~V}$ and $240 \mathrm{~V}$.

\begin{tabular}{|c|c|c|}
\hline $\mathrm{V}$ bias [V] & $200 \mathrm{~V}$ & $240 \mathrm{~V}$ \\
\hline$\sigma_{t}(N=1)$ & $34.6 \mathrm{ps}$ & $35.6 \mathrm{ps}$ \\
$\sigma_{t}(N=2)$ & $23.9 \mathrm{ps}$ & $18.0 \mathrm{ps}$ \\
$\sigma_{t}(N=3)$ & $19.7 \mathrm{ps}$ & $14.8 \mathrm{ps}$ \\
\hline
\end{tabular}

\section{Summary}

In the past 3 years we have designed, produced and tested a new type of silicon detector, characterized by a controlled, low internal gain (Low Gain Avalanche Detector). Given the low gain 


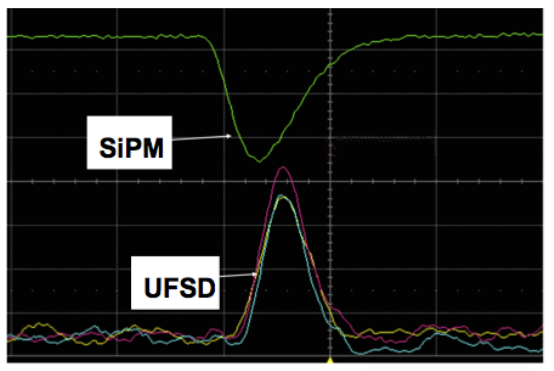

Time [ns]

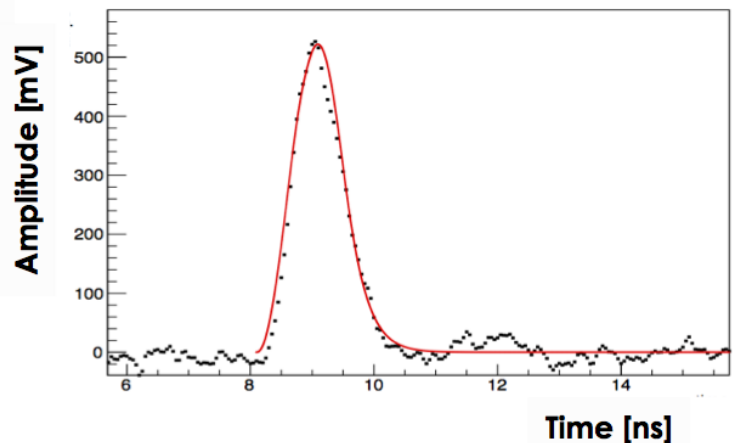

Figure 19. Left side: Signals of a beam test event showing the coincidence of 3 50-micron thick UFSD sensors and the SiPM trigger counter. Right side: Data Đ WF2 simulation (solid line).

values, these sensors are a cross-bred between traditional silicon sensors and APDs, enjoying the best characteristics of both families. The LGAD principle allowed us designing segmented silicon sensors with added timing capabilities, the so called Ultra-Fast Silicon Detectors, able of a time resolution of $\sigma_{t} \sim 35 \mathrm{ps}$. In the next years we will pursue the further development of the UFSD design, aiming at a full tracker system able to provide complete 4D information.

\subsection{Optimum Sensor Design}

Combining the information presented above, it is clear that not all geometries can be used in time tagging detectors. The most important points to have a detector with excellent time resolution are:

- High field to have saturated velocity.

- Geometries as similar as possible to parallel plate capacitors, offering uniform electric and weighting fields

- High velocity carriers, to have large $d V / d t$

- Low capacitance, to minimize jitter

- Small volumes, to minimize leakage current and therefore Shot noise

\section{Acknowledgments}

We thank our collaborators within RD50, ATLAS and CMS who participated in the development of UFSD. Our special thanks to the technical staff at UC Santa Cruz, INFN Torino, CNM Barcelona and FBK Trento. This work was partially performed within the CERN RD50 collaboration.

The work was supported by the United States Department of Energy, grant DE-FG02-04ER41286. Part of this work has been financed by the European UnionÕs Horizon 2020 Research and Innovation funding program, under Grant Agreement no. 654168 (AIDA-2020) and Grant Agreement no. 669529 (ERC UFSD669529), and by the Italian Ministero degli Affari Esteri and INFN Gruppo V. 


\section{References}

[1] G. Pellegrini et al, Technology developments and first measurements of Low Gain Avalanche Detectors (LGAD) for high energy physics applications, NIMA765 (2014) 24.

[2] H. F.-W. Sadrozinski, Exploring charge multiplication for fast timing with silicon sensors, 20th RD50 Workshop, Bari, Italy, May 30 Đ June 1, 2012.

[3] N. Cartiglia et al., Design Optimization of Ultra-Fast Silicon Detector, NIMA 96 (2015) 141-148

[4] N. Neri et al., 4D fast tracking for experiments at HL-LHC, JInst, This workshop.

[5] ATLAS upgrade project, https://cds.cern.ch/record/2055248/files/LHCC-G-166.pdf.

[6] CMS upgrade project, https://cds.cern.ch/record/2020886?ln=en\&asof=2099-12-31.

[7] HL-LHC, http://dx.doi.org/10.5170/CERN-2015-005.

[8] H. Spieler, Fast timing methods for semiconductor detectors, IEEE Transactions on Nuclear Science, Vol. NS-29, No. 3, June 1982

[9] A. Rivetti, et al., Electronics for Fast Tracking Detector, HSTD9, Hiroshima, Japan, NIMA 765 (2014) 202-208

[10] F. Cenna et al, Weightfield2: A fast simulator for silicon and diamond solid state detector, NIMA 796 (2015) 149-153.

[11] S. Ramo, Currents Induced by Electron Motion. Proceedings of the IRE 27 (9): 584-585. doi:10.1109/JRPROC.1939.228757

[12] E Martin et al., Review of results for the NA62 Gigatracker read-out prototype JInst 7 (2012) C03030

[13] M. Benoit et al., http://arxiv.org/abs/1511.04231

[14] P. Fernandez et al., Simulation of new p-type strip detectors with trench to enhance the charge multiplication effect in the n-type electrodes, NIMA 658 (2011) 98-102 .

[15] G. Pellegrini et al., Technology developments and first measurements of Low Gain Avalanche Detectors (LGAD) for High Energy Physics applications, NIMA 765 (2014) 12-16

[16] G-F Dalla Betta et al, Design and TCAD simulation of double-sided pixelated low gain avalanche detectors, NIMA 796 (2015) 154-157

[17] H.-W. Sadrozinski et al., Sensors for ultra-fast silicon detectors, NIMA 765 (2014) 7-11.

[18] H.-W. Sadrozinski et al., Ultra-fast silicon detectors, NIMA 730 (2013) 226-231.

[19] B. Baldassarriet al, Signal formation in irradiated silicon detectors, NIMA in press, doi:10.1016/j.nima.2016.06.010

[20] G. Kramberger et al., Radiation effects in Low Gain Avalanche Detectors after hadron irradiations , (JINST), 10 - 07 (2015), P07006

[21] RD50 collaboration,http://rd50.web.cern.ch/rd50/.

[22] M. Carulla et al, First 50 m thick LGAD fabrication at CNM, 28th RD50 Workshop, Torino, June 7th 2016.

[23] N. Cartiglia et al, Beam test results of a 15 ps timing system based on ultra-fast silicon detectors, submitted to NIMA, http://arxiv.org/abs/1608.08681v2. 